\title{
Metamaterials: A Leading Edge of Science and Technology
}

\author{
Vikrant Thakur \\ PG student, DIT University, Dehradun
}

\author{
Dhruva Chaudhary \\ Asst. Professor, DIT University, Dehradun
}

\begin{abstract}
This review paper is based on the brief history, classification and utilities of metamaterial in various fields. An introduction to metamaterials followed by a detailed elaboration on how to design unprecedented electromagnetic properties of metamaterials is presented. Further we discussed the different structure approaches of metamaterials and their advantages. Finally, we offer an outlook on future directions of metamaterials research.
\end{abstract}

\section{Keywords}

Metamaterials (MTMs), Left handed media (LHM), Split ring resonator (SRR), Single negative material (SNG), Double negative material (DNG), Metactronics and Electromagnetic band gap (EBG).

\section{INTRODUCTION}

The first metamaterials were developed by W.E. Kock in the late 1940s. Victor Veselago wrote an article in 1968 about the possibility of metamaterials having a negative refractive index [1]. Vaselago theoretically investigated plane wave propagation in a material whose permittivity and permeability were assumed to be negative simultaneously. He theoretically showed that for a monochromatic uniform plane wave in such a medium the direction of Poynting vector is anti parallel to the direction of the phase velocity, which was different to the case of plane wave propagation in conventional simple media. Before going in the technical aspects of metamaterials, we shall first define the meaning and scope of the word. The prefix "meta" comes from Greek and means "after" or "beyond". In English it is used to either denote that something has occurred later, derived from a Transformation, is a more highly organized or specialized form of, or transcends something else [2]. The term of metamaterial was given by Rodger M. Walser, University of Texas at Austin, in 1999. He defined metamaterials as- "Macroscopic composites having a synthetic, three-dimensional, periodic cellular architecture designed to produce an optimized combination, not available in nature, of two or more responses". Since then, many other definitions have been suggested in the literature to describe the same term. Based on Wikipedia the 'Metamaterial' is defined as-'ä material which gains its properties from its structure rather than directly from its composition'.

Due to lack of experimental verification and lack of nonexistence in nature LHM or metamaterials did not attract attention of scientific community over 30 years. The first revolution in LHM or metamerials occurred in 1996 when Pendry et all realised the artificial electric plasma using wire medium whose permittivity and permeability is negative [3]. The artificially magnetic plasma whose permeability is negative was discovered by Pendry and his co-workers in 1999 [4]. In this work, the well-known split-ring resonators (SRR) are used to achieve the magnetic response. The first artificial LHM was made by Smith et al. in 2001 using the combination of wires and SRRs [5]. In this famous experiment, the negative refraction phenomenon was verified. LHM suffers from the large loss and narrow bandwidth, despite the good properties,. Hence scientists seek other features of metamaterial beyond the negative refraction. Then the second revolution on metamaterial came in 2005 when the gradient refractive index medium was realized to bend electromagnetic waves [6], and in 2006 when the optical transformation was proposed to make invisible cloaks and to control the propagation of electromagnetic waves using metamaterials $[7,8]$.

Metamaterials, built from the molecular level up, can provide many interesting properties- Negative index of refraction, precise manipulation of waves (electromagnetic, acoustic and seismic), material chirality. Metamaterials gain their properties from structure rather than from composition. Atoms are chosen based on size and molecular ordering rather than on their macroscopic properties. Specific shape of overall structure defines the wave bending properties of the final material.

\section{METAMATERIALS CLASSIFICATION}

On the basis of $\mu$ and $\varepsilon$, material can be classified according to diagram shown in figure 1 .

\subsection{Zero Index material}

Metamaterials, in which the permittivity and/or permeability are near zero and thus the refractive index is much smaller than unity, fall at the origin of the Fig.1. These lie at the point of intersection of various types of materials.

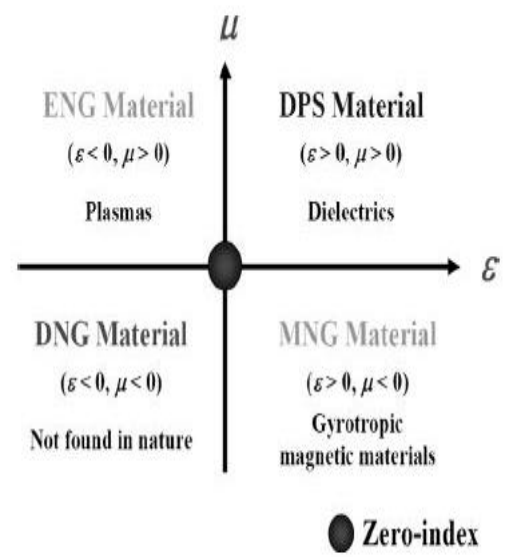

Fig 1: The nomenclature of metamnaterials, based on values of the real parts of their permittivity and permeability 


\subsection{Double positive (DPS) material}

In Fig. 1 , the first quadrant $(\varepsilon>0$ and $\mu>0)$ represents Double positive material (DPS).They are called so because both permeability and permittivity are more than zero. They are also called right-handed materials (RHM), which support the forward propagating waves.

\subsection{The permittivity/Epsilon-negative (ENZ) materials}

In Fig. 1.the second quadrant $(\varepsilon<0$ and $\mu>0)$ denotes ENZ (electric plasma), which support evanescent waves. In many metals the negative value of the electric permittivity occurs below the plasma frequency at the optical frequency range. For the frequencies lying below the plasma frequency, the real part of electric permittivity is negative and dominates the imaginary part. The geometrical parameters of the structure determine the value of plasma frequency. So by suitably adjusting the geometrical parameters of the structure, a value of negative permittivity can be achieved.

\subsection{The double negative (DNG) materials}

In the Figure 1 , the third quadrant $(\varepsilon<0$ and $\mu<0)$ denotes the left-handed materials (LHM), which was proposed by Veselago in 1968, supporting the backward propagating waves. Such materials do not occur in nature so, this class of materials has only been demonstrated with artificial constructs. The DNG meatmaterials also show negative refraction properties. Ability to provide phase compensation or phase conjugation due to their negative refraction is one of the interesting features of DNG media. Because of its dispersive nature the DNG medium, might also be used for time-domain applications as an effective dispersion compensation device.

\subsection{The permeability/Mu-negative (MNG) metamaterials}

The fourth quadrant $(\varepsilon>0$ and $\mu<0)$ represents magnetic plasma, which supports evanescent waves. When this property is realized in metamaterial design it is known as $\mu$ negative metamaterial. These metamaterials are made from SRR's. It is the metal split ring resonator structure (SRR) shown in figure 3. It was first proposed in [8]. It is basically a sub wavelength magnetic resonator made of inductive metallic rings loaded with capacitive gaps if the excitation magnetic field $\mathrm{H}$ is perpendicular to the plane of the rings, so as to induce circulating current in the rings, which in turn give rise to a magnetic dipole moment.

\section{DESIGNING OF METAMATERIALS}

Sub wavelength resonators of various types, forming building blocks for metamaterial are introduced in the past years, as for example: thin wires [9], [14], Swiss rolls [9], SRRs [10], electric SRRs (eSRRs) [15], [16], pairs of rods [11], [12], [17], pair of crosses [18], fishnets [13], [19],etc. Some of them display negative refractive index while, some of them are designed for either magnetic or electric response. The basic structures of SRRs and thin wires are sufficient to achieve the required electromagnetic properties at frequencies below infrared.

\subsection{Lattice of Thin Wires}

The $\varepsilon$ negative metamaterials are realised from this structures. At high frequencies, i.e.at the visible or UV band for metals, the electric response of natural conductive materials typically takes place. This is evident from the electric plasma frequency, $\boldsymbol{w}_{\mathrm{ep}}$, which can be formulated as

$$
w_{e p}^{2}=\frac{n e^{2}}{\varepsilon_{o m_{e f f}}}
$$

$\boldsymbol{n}=$ electron density, $\boldsymbol{\varepsilon}_{\boldsymbol{o}}=$ vaccum permittivity, $\boldsymbol{m}_{\text {eff }}=$ electron effective mass, $\boldsymbol{e}=$ electron charge.

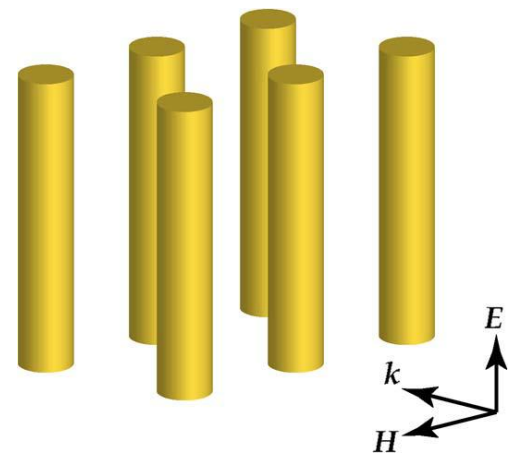

Fig 2: Lattice of thin metallic wires

The plasma frequency must be modified in order to achieve an electric response at a lower frequency range e.g. microwave region. In the equation (1) through changes in the electron density and electron mass the plasma frequency must be reduced.

A metamaterial structure made up of thin metallic wires, similar as shown in fig. is a workable solution [20]. The structure consists of thin parallel conducting wires. When excitation electric field is parallel to the axis of the wire so as to induce a current along them and generate equivalent electric dipole moment. From the analysis, given that a is the lattice spacing and $r$ is the wire radius, the plasma frequency of the structure now becomes

$$
w_{e p}^{2}=\frac{2 \pi c_{0}^{2}}{a^{2} \operatorname{In}\left(\frac{a}{r}\right)}
$$

The structure can be characterised by an effective permittivity that takes on a Drudes model, by assuming infinite length wire.

$$
\varepsilon_{\text {eff }(w)=1-} \frac{w_{e p}^{2}}{w^{2}+j 2 w}
$$

Where is responsible for the propagation loss. At $w<w_{e p}$ the permittivity becomes negative.

\subsection{SRRs}

A unit cell of the original double SRRs is basically composed of two concentric metallic rings with opposite splits or gaps. A current flow is established by the rings, which further builds a magnetic dipole parallel or anti parallel to the magnetic to the magnetic fields when the SRR is coupled to a magnetic field component oscillating in the axial direction. The loop inductance and gap capacitance are equivalent to an LC resonant circuit, causing a strong magnetic response at its resonance. The resonance frequency is lowered since the inner concentric ring contributes to the net capacitance of the double SRR. The ratio between the operating wavelength and lattice constant is hence boosted up by the ring, making the SRRs appear more homogenous to the electromagnetic excitations. Inspite of this, the inner ring can be removed without a significant impact on the SRRs function while shifting the resonance frequency. Lorentzian model describes 
a metamaterial structure composed of periodically aligned SRRs under a magnetic excitation by an effective magnetic permeability [21]

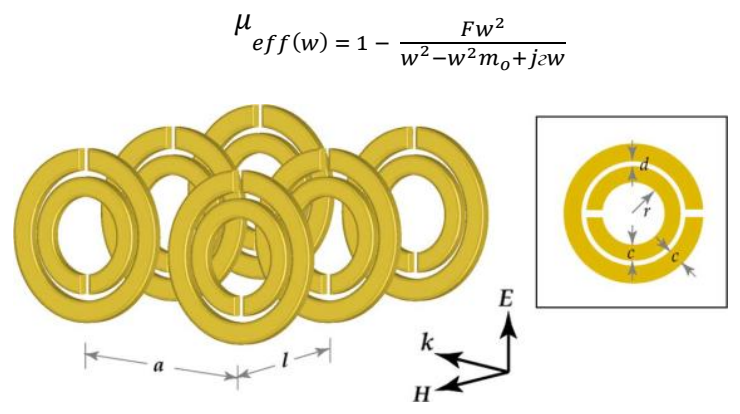

Fig 3: Double SRRs

Where $\mathrm{w}_{\mathrm{mo}}$ is the magnetic resonance frequency, $\Gamma$ represents the energy dissipation, and F is the fill factor of the SRR. The magnetic resonance frequency and the SRRs geometry are related with each other through [21].

$$
w^{2} m_{o=} \frac{3 I c_{0}^{2}}{\pi \ln \frac{2 c}{d} r^{3}}
$$

The magnetic plasma frequency is the frequency where the permeability crosses zero and is given by

$$
w_{m p}^{2}=\frac{w_{m o}^{2}}{(1-F)}
$$

The SRRS have a positive response to the magnetic fields at frequencies lower than resonance and the response becomes negative between the resonance and plasma frequency. This structure supports paramagnetism $\quad\left(\mu_{\text {eff }}>1\right)$ and diamagnetism $\left(\mu_{\mathrm{eff}}<1\right)$, including a negative permeability.

\subsection{SRRs/Wires Combination}

The $1^{\text {st }}$ DNG metamaterial realised by Smith et al. in 2000 was actually a combination of the thin wires based ENG structure and the SRR based MNG structure [22]. It was assumed that the new composite material exhibited a macroscopic permittivity equal to that of the thin wire ENG medium and a macroscopic permeability equal to the permeability of the SRR based MNG medium. In the Fig.4 is shown such a combination of SRR and wires.

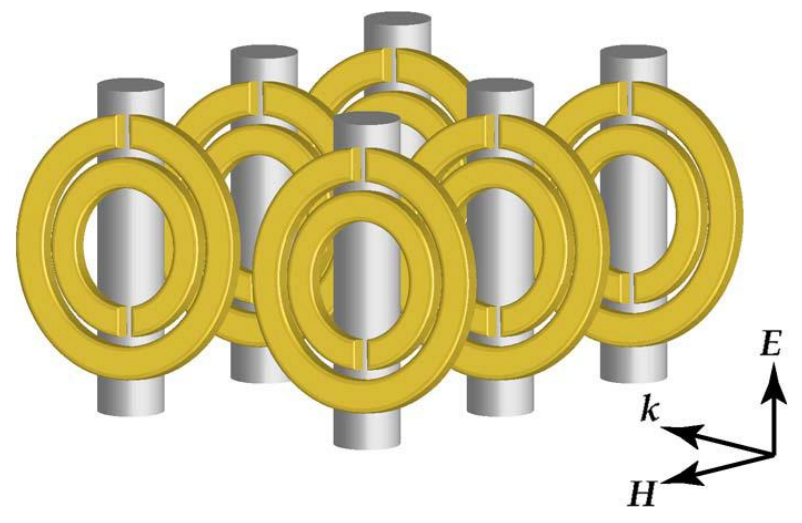

Fig 4: SRRs/Wires Combination

\section{APPLICATIONS OF METAMTATERIAL}

\subsection{Superlenses}

Super lens or perfect lens is an optical lens with resolution capabilities that go significantly beyond ordinary lens. It is able to do this by beating the diffraction limit that conventional lenses have, by using a metamaterial with negative index of refraction. Standard diffraction theory imposes a resolution limit when imaging using conventional lenses. This fundamental limit is called diffraction limit, is accredited to finite wavelength of the electromagnetic waves. In 2000, John Pendry extended the analysis of Veselago's lens to include evanescent waves and observed that such lenses could overcomethe classical diffraction limit. The first super lens in the microwave regime was realized in 2004 [23], which demonstrated resolution three times better than the diffraction limit. Later Fang et. al, proposed the first optical super lens using thin silver film [24] which break the diffraction limit and produce sup er resolution images [25]. In 2010 a nano wire array proto type described as a three dimensional metamaterial nano lens [26]. Super lenses find great application in super resolution medical imaging, optical imaging, and non destructive detection. The big obstacle of superlens in applications is the loss.

\subsection{Cloaking Devices}

Cloaking devices were investigated in the late 1980s and early 1990s [27].For general meatmaterials, the cloaking devices have attracted more and more attention. Metamaterials have provided basis to realize a practical cloaking device by successful demonstration of invisible cloaks experimentally in the microwave regime $[28,29]$. The idea was first published by Prof. Sir John Pendry in 2006. Transformation optics is a novel method for the design of invisibility cloak-a-material that can be used to hide the object from detection [30,31].If an object is covered with such a metamaterial then that will mean that light waves will flow around the object and hence, will not be detected. In 2009,a group of scientists announced cloaking at optical frequencies. In this case the cloaking frequency was centred at $1500 \mathrm{~nm}$ (in infrared region) [32].The metamaterial acoustic cloak is designed to hide objects submerged in water. Cloaking devices find their main application in stealth technology.

\subsection{Antenna Technology}

There are class of antennas which use metamaterials for increasing the overall performance of electrically small antennas. Metamaterial based antennas exhibit several other useful properties such as high directivity, improved efficiency-bandwidth performance, stepping up of radiated power etc. [33, 34]. Metamaterials when used in the ground planes, surrounding the antennas, offer improved isolation between radio frequency or microwave channels (MIMO) antennas arrays. They have also been used to increase the beam scanning range in leaky wave antennas by using both the forward and backward waves. Various metamaterial antennas systems have been employed to support surveillance sensors, communication wires, high frequency battlefield communication, navigation systems and control systems [35, 36].

\subsection{Absorbers}

A metamaterial absorber is a type of metamaterial intended to efficiently absorb electromagnetic radiation such as light. Metamaterial absorbers, manipulate the loss components of metamaterial permittivity and magnetic permeability, to absorb large amount of electromagnetic radiation [37]. The metamaterial utilizes tiny geometric shapes to absorb both the electrical and magnetic properties of electromagnetic waves. Boston College - designed "perfect absorber of light" with metamaterials. This property of metamaterials can be 
incorporated into solar cells to enhance sensitivity for greater energy conversion. Future applications of metamaterial absorbers include emitters, spatial light modulators, camouflage and use in thermo photovoltaics.

\subsection{Biosensors}

Biosensors are essential in many areas, such as disease diagnostics, environmental monitoring, and food safety, and they are also vital tools in the investigation of biological phenomena. Fluorescence-based methods have proven useful in analyzing both genomic and proteomic microarrays [38, 39] and in imaging, including single molecule detection inside living cells [40,41]. Recently, biosensing technologies based on metamaterials have attracted significant attentions from the microwave to optical frequency because of their cost-efficient and label-free biomolecule detection, the metamaterial-based sensors are classified into three types according to operating frequency of sensing biomolecule and component: microwave biosensor, terahertz biosensor and plasmonic biosensor.

\section{RESEARCH AREAS OF METAMATERIAL}

\subsection{Seismic metamaterials}

Seismic metamaterials are metamaterials which are designed to neutralize the adverse effects of seismic waves on artificial structures, which exist on or near the surface of the earth.[42][43][44] For seismic metamaterials to protect surface structures, the proposal includes a layered structure of metamaterials, separated by elastic plates in a cylindrical configuration. Seismic metamaterial have the ability to manipulate seismic waves which are created by earthquake. the idea is proposed that if the metamaterial used as ring around a building foundation then there have possibility that it diverts the most destructive seismic waves around the entire building [45], [46].

\subsection{Acoustic Metamaterials}

Acoustic metamaterials are artificially fabricated materials designed to control, direct and manipulate sound in the form of sonic waves. Sonic waves may now be extended to negative refraction domain. These can be applied to superior lenses for ultrasound machines. Walls of the material could be built to soundproof houses or it could be used in concert halls to enhance acoustics or direct noise away from certain areas. These also find applications in military may to conceal submarines from detection by sonar and to create a new class of stealth ships [47].

\subsection{Tunable Metamaterials}

Regular metamaterial only respond to one frequency or frequency band contrary to that tunable metamaterial has the ability to randomly adjust frequency change in the refractive index. Electromagnetic band gap (EBG), also known as photonic band gap (PBG), and negative refractive index material (NIM) are the ongoing research area of this domain. Scientists are developing a new metamaterial design which allows will show the switching property. The device can switch from a normal flat mirror to a focusing/defocusing mirror without changing its shape [48], [49]. Tunable metamaterials uses an anisotropic liquid crystal to allow frequency tuning. These metamaterials find application in several electronically tuned microwave systems in defence, space and commercial communications applications.

\subsection{Metactronics}

Metactronics is an approach based on metamaterial for designing future electronics on a nano scale. The unique light-properties of metamaterials enable use of light in designing electronic circuitry faster and more precisely. This concept was given by the professor Nader Engheta in 2007. Metactronics provide a mechanism for tailoring, patterning and manipulating optical electric fields. Nano particles will play the role of lumped nanocircuit elements. These optical nano electronics will work with light instead of electron transport [50].

\subsection{Power Harvesting Metamaterial}

Metamaterials are well-suited for electromagnetic power harvesting. Power harvesting devices convert one type of energy to another. Researchers say the power-harvesting metamaterial could potentially be built into a cell phone, allowing the phone to recharge wirelessly while not in use. This feature could, in principle, allow people living in locations without ready access to a conventional power outlet to harvest energy from a nearby cell phone tower instead. Improve the energy efficiency of appliances by wirelessly recovering power that is now lost during use [51], [52].

\section{CONCLUSION}

All experimental and theoretical demonstration of metamaterials shows that metamaterials have become an extremely fertile research area. The super resolution imaging, the nanostructure metamaterials or metactronics, light harvesting for improved solar cell technology and power harvesting are the modern trend of metamaterial applications. The unique electromagnetic properties provided by metamaterials attract considerable attention of researchers from multiple disciplines. Many other fascinating discoveries and applications are waiting for us to explore.

\section{REFERENCES}

[1] V. G. Veselago, "The electrodynamics of substances with simultaneously negative values of epsilon and mu." Soviet Physics Uspekhi,vol. 10, no. 4, pp. 509-514, 1968.

[2] Merrian- Webster Collegiate Dictionary $10^{\text {th }}$ Edition, Massachusetts, pp.730, 1997

[3] Pendry, J.B., A.J., Stewart, W.J., Ypungs, I: Extremely low frequency plasmons in metallic mesostructure, Phys. Rev. Lett. 76, 4773-4776, 1996.

[4] Pendry, J.B., Holden, A.J., Robbins, D.J., Stewart, W.J; Magnetism from conductors and enhanced non linear phenomenon. IEEE Trans. Micro Theory Tech. 47, 20752084, 1999

[5] Leonhardt, U.: Optical conformal mapping Science 312, 1777,2006

[6] Jiang, W.X., Cui, T.J., Chin, J.Y., Chang, Q., Yu, G.X., Yang, X.M, Liu, R.: Equal thickness invisible cloaks for arbitrary polygonal - cylindrical regions. Eur Phys. J. Appl. Phys 46, 20702,2009

[7] Kwon, D.H., Werner, D.H.: Transformation optical designs for wave collimators, flat lenses and right angle bends. New J. Phys. 10. 115023, 2008

[8] Leonhardt, U,: Notes on conformal invisibility devices. New J. Phys.8, 118, 2006 
[9] R.M. Walser, 'Electromagnetic metamaterials, "in Proc SPIE - Complex Medium 11; Beyond Linear Isotropic Dielectrics, 2001, vol. 4467,pp 1-15

[10] Smith, D.R., Mock, J.J., Starr, A.F., Schurig, D.: Gradient index metamaterials. Phys. Rev. E, vol. 71, Issue. 3, pp. 036609, 2005

[11] J. B. Pendry, A. J. Holden, W. J. Stewart, and I. Youngs, BExtremely low frequency plasmons in metallic mesostructures,[ Phys. Rev. Lett., vol. 76, no. 25, pp. 4773-4776, Jun. 1996.

[12] J. B. Pendry, A. J. Holden, D. J. Robbins, and W. J. Stewart, BMagnetism from conductors and enhanced nonlinear phenomena,[ IEEE Trans. Microw. Theory Tech., vol. 47, no. 11, pp. 2075-2084, Nov. 1999.

[13] D. A. Powell, I. V. Shadrivov, and Y. S. Kivshar, BCutwire-pair structures as two-dimensional magnetic metamaterials,[ Opt. Lett., vol. 16, no. 19, pp. 15 185-15 190, Sep. 2008.

[14] G. Dolling, C. Enkrich, M. Wegener, J. F. Zhou, C. M. Soukoulis, and S. Linden, BCut-wire pairs and plate pairs as magnetic atoms for optical metamaterials,[ Opt. Lett., vol. 30, no. 23, pp. 3198-3200, Dec. 2005.

[15] G. Dolling, M. Wegener, C. M. Soukoulis, and S. Linden, BNegative-index metamaterial at $780 \mathrm{~nm}$ wavelength, [Opt. Lett., vol. 32, no. 1, pp. 53-55, Jan. 2007.

[16] J. B. Pendry, A. J. Holden, D. J. Robbins, and W. J. Stewart, BLow frequency plasmons in thin-wire structures,[ J. Phys., Condens. Matter, vol. 10, no. 22, pp. 4785-4809, Jun. 1998.

[17] D. Schurig, J. J. Mock, and D. R. Smith, BElectric-fieldcoupled resonators for negative permittivity metamaterials,[ Appl. Phys. Lett., vol. 88, no. 4, pp. 041109-1-041109-3, Jan. 2006.

[18] W. J. Padilla, M. T. Aronsson, C. Highstrete, M. Lee, A. J. Taylor, and R. D. Averitt, BElectrically resonant terahertz metamaterials: Theoretical and experimental investigations,[ Phys. Rev. B, Condens. Matter, vol. 75,no. 4, pp. 041102-1-041102-4, Jan. 2007.

[19] V. Shalaev, W. Cai, U. Chettiar, H. Yuan, A. Sarychev, V. Drachev, and A. Kildishev, BNegative index of refraction in optical metamaterials,[ Opt. Lett., vol. 30, no. 24, pp. 3356-3358, Dec. 2005.

[20] C. Imhof and R. Zengerle, BPairs of metallic crosses as a left-handed metamaterial with improved polarization properties,[ Opt. Express, vol. 14, no. 18, pp. 8257-8262, Sep. 2006.

[21] S. Zhang, W. Fan, N. C. Panoiu, K. J. Malloy, R. M. Osgood, and S. R. J. Brueck, BExperimental demonstration of nearinfrared negative-index metamaterials,[ Phys. Rev. Lett., vol. 95, no. 13, pp. 137 404-1-137 404-4, Sep. 2005.

[22] J. B. Pendry, A. J. Holden, W. J. Stewart, and I. Youngs, BExtremely low frequency plasmons in metallic mesostructures,[ Phys. Rev. Lett., vol. 76, no. 25, pp. 4773-4776, Jun. 1996.

[23] J. B. Pendry, A. J. Holden, D. J. Robbins, and W. J. Stewart, BMagnetism from conductors and enhanced nonlinear phenomena,[ IEEE Trans. Microw. Theory Tech., vol. 47, no. 11, pp. 2075-2084, Nov. 1999.

[24] D. R. Smith, W. J. Padilla, D. C. Vier, S. C. NematNasser, and S. Schultz, BComposite medium with simultaneously negative permeability and permittivity, [ Phys. Rev. Lett., vol. 84, no. 18, pp. 4184-4187, May 2000.

[25] Grbic,A.Eleftheriadesm.G.V :Overcomimg the diffraction limit with a planar left-handed transmissionline lens.Phys.Rev.Lett.92,p.117403,2004.

[26] Fang,N.et all,'Sub-diffraction limited optical imaging with a silver superlens, 'Science,vol.308,no.5721,pp.534537,2005 .

[27] Igor I., Smolyaninov, Yu-Ju Hang,Christopher C.Davis, "Magnifying superlens in the visible frequency range", Science vol 315, no.5819,pp 1699-1701,2007.

[28] B.D.F.Casse,W.T.lu,Y.J.Huang,E.Gultepe,L.Menon, S.Sridhar,"Super-resolution imaging using a 3-D metamaterial nanolens," Applied Physics Letters ,vol .96,no 023114,pp 1-3,2010.

[29] E.Wolf and T.Habashy, "Invisible bodies and uniqueness of the inverse scattering problem," J.Mod. opt.40

[30] Schurig,D.,Mock,J.J,Justice,B.J.,et al.: Metamaterial electromagnetic cloak at microwave frequencies science 314,977-980,2006

[31] Liu,R.,Ji,C.,Mock,J.J.,Chin,J.Y.,Cui,T.J.,Smith.D.R.:Bro adband ground plane cloak.Science 323,366(2009)

[32] J.B.Pendry,D.Schurig and D.R.Smith,"controlling electromagnetic fields," science,vol.312,pp.1780 1782,June 2006.

[33] D.Schurig,J.J.Mock,B.J.Justice,S.A.Cummers,J.B.Pendry ,A.F.Star and D.R.Smith." Metamaterials electromagnetic cloak at microwave frequencies ," science,vol.314,pp.977-980,November 2006.

[34] Lucas H.Gabrielle, Jamie Cardenas, Carl B. Poratas, Michal Lipson, "Silicon nanostructure cloak operating at optical freq.," Nature Photonics, vol.3, pp.461-463, 20 July 2009.

[35] R.W. Ziolkowski and A. Kipple, "Application of double negative metamaterials to increase the power radiated by electrically small antennas," IEEE Trans. Antennas Propag., vol.51, pp. 2626-2640, Oct. 2003.

[36] R.W. Ziolkowski and A. Erentok, "Metamaterial-based efficient electrically small antennas," IEEE Trans. Antennas Propag., vol.54, no.7, pp. 2113-2130, July 2006.

[37] Fangming Zhu,Qingchun Lin, Jun Hu, "A Directive Patch Antenna with a Metamaterial Cover," Asia Pacific Microwave Conference Proceeding volume 3. Suzhou china 2005.

[38] Rui Wang, Bo Yuan, Gaofeng Wang, Fan Yi (2007). "Efficient Design of Directive Patch Antennas in Mobile Communications Using Metamaterials," International Journal of Infrared and Millimeter Waves, vol. 28, issue. 8, pp. 639-649, 2007.

[39] http://en.m.wikipedia.org/wiki/metamaterial_absorbser 
[40] Sanders G.H.W., Manz A. Chip-based microsystems for genomic and proteomic analysis. Trends Anal. Chem. 2000; 19:364-378.

[41] Solinas Toldo S., Lampel S., Stilgenbauer S., Nickolenko J., Benner A., Dohner H., Cremer T., Lichter P. Matrixbased comparative genomic hybridization: Biochips to screen for genomic imbalances. Genes Chromosomes Cancer. 1997; 20:399-407. [PubMed]

[42] Michalet X., Kapanidis A.N., Laurence T., Pinaud F., Doose S., Pflughoefft M., Weiss S. The power and prospects of fluorescence microscopies and spectroscopies. Annu. Rev. Biophys. Biomol. Struct.2003;32:161-182. [PubMed]

[43] Webb S.E.D., Roberts S.K., Needham S.R., Tynan C.J., Rolfe D.J., Winn M.D., Clarke D.T., Barraclough R., Martin-Fernandez M.L. Single-molecule imaging and fluorescence lifetime imaging microscopy show different structures for high- and low-affinity epidermal growth factor receptors in A431 cells. Biophys. J. 2008;94:803819. [PMC free article] [PubMed]

[44] Johnson, R. Colin (2009-07-23). "Metamaterial cloak could render buildings 'invisible' to earthquakes". EETimes.com. Retrieved 2009-09-09.

[45] Barras, Colin (2009-06-26). "Invisibility cloak could hide buildings from quakes". New Scientist. Retrieved 2009$10-20$.

[46] Brun, M.; S. Guenneau, and A.B. Movchan (2009-0209). "Achieving control of in-plane elastic waves". Appl.
Phys. Lett. 94 (61903):1-7. arXiv:0812.0912.Bibcode: 2009ApPhL.. 94f1903B. doi: 10.1063/1.3068491.

[47] Johnson, R. Colin (2009-07-23). "Metamaterial cloak could render buildings 'invisible' to earthquakes". EETimes.com. Retrieved 2009-09-09.

[48] Barras, Colin (2009-06-26). "Invisibility cloak could hide buildings from quakes". New Scientist. Retrieved 2009$10-20$.

[49] http://en.m.wikipedia.org/wiki/Acoustic_metamaterials

[50] http://www.pratt.duke.edu/news/wireless-deviceconverts-lost-energy-electric-power.

[51] Allen M.Hawkes, Alexander R.katko and Steven A.Cummer, "A microwave metamaterial with integrated power harvesting functionality," Applied Physics Letters, vol.103, pp. 163901, october2013

[52] Nader engheta, "Circuits with Light at Nanoscales: Optical Nanocircuits Inspired by Metamaterials," Science, Vol. 317, no. 5845, pp. 1698-1702, 21 September 20

[53] llya V. Shadrivov, Polina V. kapitanova, Stanislavi. Maslovski and Yuris. Kivshar, "Metamaterials controlled with light," Phys. Rev. Lett., vol. 109, pp. 083902-1083902-4 , 2012

[54] http://en.wikipedia.org/wiki/Tunable_metamaterials\#cite _note-Lapine-structure-2. 